\title{
Optimization of damping unit
}

\author{
J. Kolář \& K. Adámek \\ VÚTS Liberec, Czech Republic
}

\begin{abstract}
This paper reports the numerical investigation and optimization of a damping unit where oil flow interacts with an elastic chocking plate. Chocking of flow from a by-pass valve is the basic principle of hydraulic shock absorbers. However, cavitation phenomenon caused by strong shocking occurs at high frequencies of an actuating movement and the original damping unit characteristic is being distorted. We used the computational fluid dynamic to minimize the distortion of characteristic as much as possible. To satisfy the high number of unsteady simulations, the involved fluid-structure interaction needs to be coupled in a different way than the routine is. The plate deformation is coupled with an average hydrodynamic loading pressure by a single aforecomputed third polynomial equation being predicted by FEM. Numerical simulations of flow by the use of FVM exploiting dynamic mesh in Fluent commercial code then provides a flow field pattern and loading pressure within the unit itself. A User Defined Function (UDF) enables the deformation of the domain boundaries in a coupled way. Finally, the time dependency of the plate position and its dynamic behaviour is obtained. Highly dynamic manners appeared and numerous techniques providing a better stability of computations had to be investigated and developed. The state where the most cavity bubbles are being induced is further applied to shape optimization at the steady state. The shape-based similarity optimization of by-pass geometry is then carried out to minimize the formation of cavitation bubbles.
\end{abstract}

Keywords: damping unit, shape optimization, computational fluid dynamics, chocking plate, cavitation, user defined functions, shape based similarity.

\section{Introduction}

The movement of a thin spring-steel plate induced by the impact of oil flow is an example of fluid-structure interaction. This interaction can be found in many engineering devices. The mentioned case is a typical one in the field of chocking 
fluids. The originally stiff plate is loaded and deformed by the surrounding flow. Consequently, the chocking plate truckles to loading and gets deformed. In computational fluid dynamics (CFD), the number of iterative steps is needed to solve the steady state of oil flow and the resulting plate shape which corresponds one to another. In our case, oil flow is induced by the sinusoidal movement of the unit walls with respect to the relation that follows

$$
y^{+-}=y_{0} \pm A \cdot \sin (2 \cdot \pi \cdot f \cdot t)
$$

For the device scheme, see Figure 1 . Finally the task is necessarily unsteady with a frequency of $\mathrm{f}=2 \mathrm{~Hz}$.

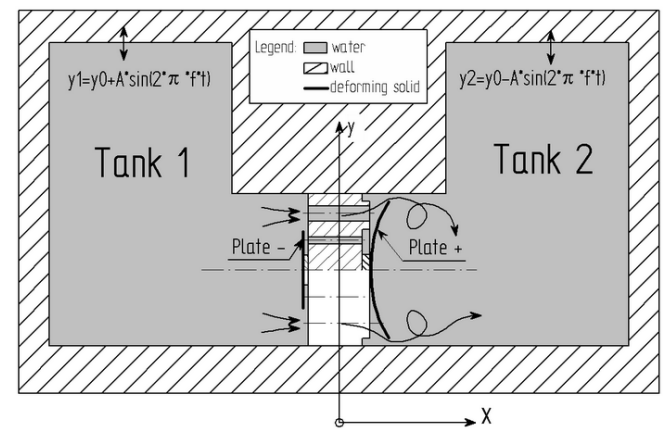

Figure 1: $\quad$ Scheme of damping unit with unsteady movement relations.

We decided to apply the simplified, but more efficient approach to get into the solved problem because the conventional approach with intercommunication and data transfers between FVM and FEM solvers gains unacceptable time cost for unsteady simulations. The non-conventional approach of the mentioned task includes the number of applied techniques and requirements such as dynamic domains, self-guarding routines, and deforming functions.

\section{Unsteady fluid-structure interaction and cavity identification}

\subsection{Fluid-structure coupling}

The dynamic computational domain feature allows the computational domain to change its shape with conserved topology with respect to the prescribed relation which relates the surface loading and structure stiffness. As a result, unsteady simulations are not composed from the set of solutions of segregated domains being solved from initialization. Instead, the dynamic solution of the initial domain which underlies the deformation is performed. The computational grid changes upon the predicted shape which results from pressure load at the deformed boundary. More detailed view can be taken in [1] and [2]. The predicted shape, shown in Figure 2(a) is afore-computed by FEM analysis in Mark 3D software package. The surface of the observed plate is one-sidedly loaded by substitutive pressure. This approach presumes minimal or even 
negligible twist deformation. This condition is being met as tested in numerous cases. The plate is made of spring steel 12090 with $\mathrm{R}_{\mathrm{m}}=950 \mathrm{Mpa}$ and $\mathrm{E}=2.171 \cdot 10^{11} \mathrm{~Pa}$ and fully fixed all-around the inner diameter-r. The shapesubstitutive cubic polynomial is derived from the predicted deformation by the method of least squares employing Gauss elimination. The local deformation of the boundary node at the radial coordinate $\varphi_{j}=R-r_{j}$, is then given by an average loading pressure given as

$$
p_{\text {avg }}=\frac{i \sum_{i=0}^{i=N^{+}} F_{i}^{+}+\sum_{i=0}^{i=N^{-}} F_{i}^{-}}{\left(\sum_{i=0}^{i=N^{+}}-A[0]_{i}^{+}+\sum_{i=0}^{i=N^{-}}-A[0]_{i}^{-}\right) / 2}
$$

and as a relation which follows

$$
\Delta x_{j}\left(r_{j}, p_{\text {load }}\right)=\frac{-0.0032 \cdot r_{j}{ }^{3}+0.066 \cdot r_{j}^{2}+0.0654 \cdot r_{j}-0.0163}{16 \cdot 10^{5}} \cdot p_{\text {load }}
$$

Due to the fluid-structure interaction, the plate position always affects the surrounding flow field. This has an effect of overestimated plate position predicted. We have tested and applied absorber coefficient $\mathrm{k}$ which corrects the increase of the actual plate position defined as

$$
\Delta x^{\prime}{ }_{j}=\Delta x_{j} * k \text { where } k \in R \mid 0<k<1 .
$$

The influence of absorption coefficients may be studied in Figure 2(b).
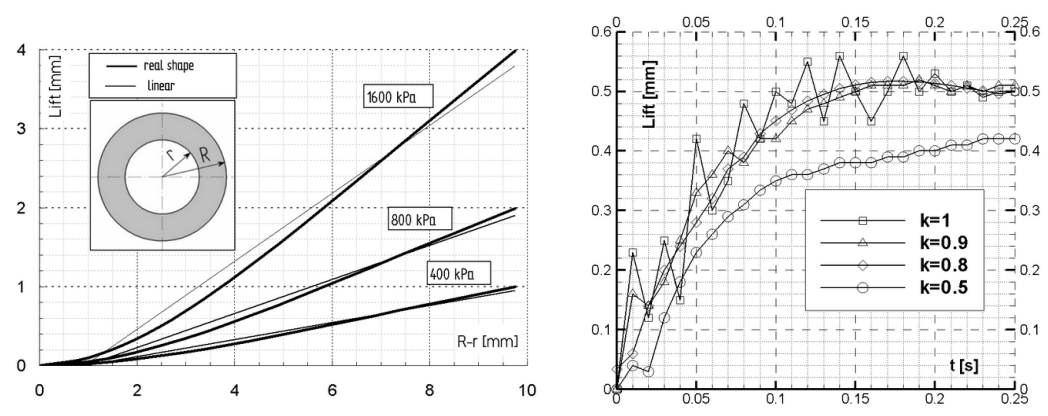

Figure 2: Deformed plate shape predicted by FEM (a) and the overestimation of plate position with various absorption coefficients (b).

The underestimation of the total plate position by coefficient $\mathrm{k}$ is limited by the differential approach. A new plate position is based on the actual position and computed increase of coordinate corrected by $\mathrm{k}$. The dynamic movement of the plate was observed even when the nonsensical movements were smoothed out. This led to a large deformation of grid elements or even to a formation of negative cell volume. To avoid this, the self-guarding routine was implemented into the source code. The routine of the source code supervises the maximal increase of coordinate available at the base of the quality of boundary adjacent 
cells. The ultimate angular node displacement is established from the equation (5). For the scheme of approach, see Figure 3. More detailed expression can be found in [3].

$$
\operatorname{tg}\left(d \alpha_{\max }\right) \cong \min \left\{\frac{3 \cdot \vec{d} r[0]_{i}}{r_{\max , c}}, \ldots, \frac{3 \cdot \vec{d} r[0]_{N}}{r_{\max , N}}\right\}
$$

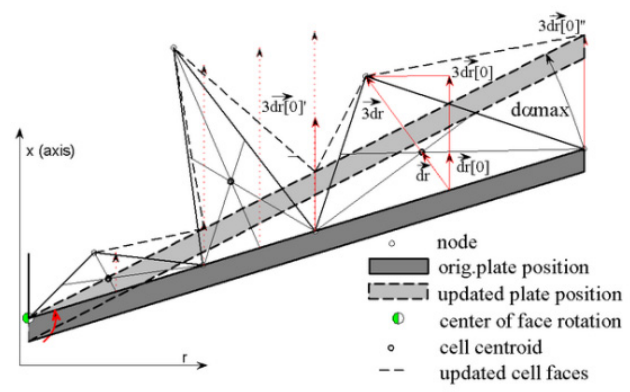

Figure 3: $\quad$ Scheme of ultimate angular displacement based on equation (5).

\subsection{CFD simulations}

We have used the commercial CFD package Fluent version 6.3 to solve the 3D RANS equations for the incompressible oil phase, with a time step 1E-5s by the final volume technique. The model of the domain consists only from a period of one twelfth of the real device. The turbulence model k- $\varepsilon$ Standard completes the system of governing equations [4]. For all equations, convective terms are discretized using a second-order upwind scheme except the linear one for pressure. The discretized system is solved in a simple way. A pressure based, first order implicit solver has been used for unsteady formulation when dynamic mesh applied. A cavitation phenomenon is enabled by the secondary phase defined as incompressible oil vapour with a saturated pressure of $6 \mathrm{kPa}$ [4]. The criterion for assessing convergence was based on the root mean square of the density residues expressed by

$$
R(\xi)=\left[\sum_{i=1}^{M}\left(\frac{\partial \xi}{\partial t}\right)_{i}^{2}\right]^{\frac{1}{2}}
$$

where $\mathrm{M}$ is the number of grid points and $\xi$ is the variable considered to check (mass, energy, momentum, etc.). Generally, the next time step is taken when residuals fall below $1 \mathrm{E}-4$.

\subsection{Results of unsteady fluid-structure interaction}

For brief examples of results, see Figures 4 and 5. Axial and radial coordinate $x$, $\mathrm{r}$ and angular coordinate fi_y define the positions of pressure minima responsible for cavitation within time period. See Figure 4(a). To get easier in the task, see Figure 4(b). 

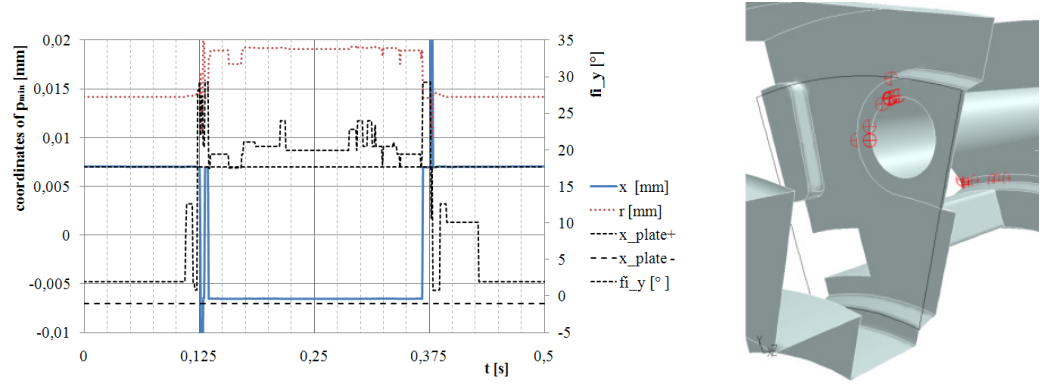

Figure 4: Spatial localization of pressure minima $\mathrm{p} \_\min (\mathrm{x}, \mathrm{r}, \mathrm{fi} \mathrm{y})$ (a) and its $3 \mathrm{D}$ visualization in various time steps for $\overline{\mathrm{f}}=2 \mathrm{~Hz}(\mathrm{~b})$.
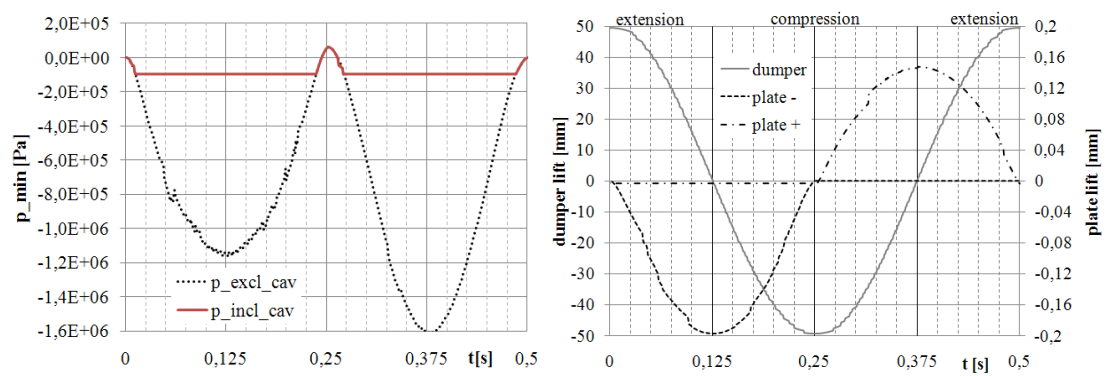

Figure 5: Temporal localization of pressure minima (a). Affected plates lift (b).

There is no temporal point during the damper lift significantly more responsible for the formation of cavitation bubbles as can be seen in Figure 5 a). The level of minimum pressure follows the actual oil flow velocity until the saturation pressure is achieved. From the case with cavitation excluded, the consequent smooth tendency of pressure minima can be observed (even if the result is not fully physically meaningful). Plate lift at the outer diameter $\mathrm{R}$ is recorded and is to be seen in Figure 5b).

\section{Steady optimization}

\subsection{Method of by-pass channel and chocking plate optimization}

We tried to find the combination of by-pass channel shape and plate stiffness with conserved pressure jump to minimize the production of cavity bubbles and we have decided to apply the optimization procedure to the stationary state adequate to $\mathrm{t}=0.05 \mathrm{~s}$ from the bottom dead centre. This state is of fully developed cavitation flow with an acceptable mass flow rate through the valve, not very threatening for the numerical stability of simulations itself. For the optimization itself, the shape based similarity optimization developed and described in [1] was applied. The shape of the by-pass nozzle is given by the cubic spline, 
computed simultaneously from the set of three given parameters $\mathrm{y}_{1}, \mathrm{y}_{2}=\mathrm{y}_{0}$ and $\alpha_{2}$, see Figure 6 . We have applied the ordinary Gauss's elimination method to find out the spline coefficients. The fourth parameter is the plate lift, used to adjust the oil mass flow rate to the value being equal to the real mass flow at $0.05 \mathrm{~s}$. The iterative adjustment of plate lift is provided by simple Newton's gradient method with maximum six steps. An average error of the plate position after six steps does not exceed 1e-5 $\mathrm{m}$ in test cases, which is sufficient for the solved case. We have applied the dynamic mesh model update to derive the most benefits of the minimal deformation path given by the shape-based similarity. A User Defined Functions (UDF) described in [3] and applied by Koláŕ and Dvorák in [2] is compiled to drive the wall boundary deformation over the whole sequence of nozzle shapes (vectors). The original domain that underlies the deformation has a simple linear shape. UDF updates the $\mathrm{y}$-coordinate $\left(\mathrm{y}_{\text {node }}\right)$ of each node at time $t+1$ (new shape) according to the spring based deformation defined as follows

$$
y_{\text {node }}^{t+1}=\frac{y_{\text {node }}^{t}}{y_{\text {wall }}^{t}} y_{\text {wall }}^{t+1}
$$

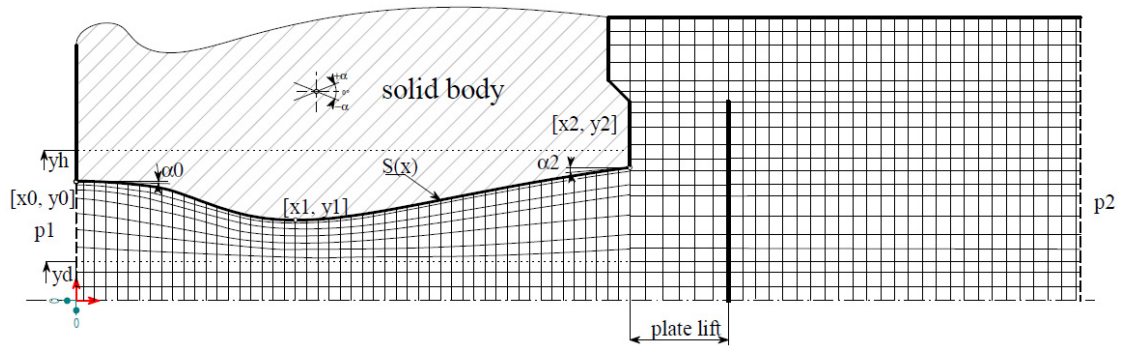

Figure 6: Scheme of domain for optimization of channel shape and chocking plate stiffness. Driving parameters of spline: $y 1, y 0=y 2, \alpha 2$ and boundary conditions.

Optimization of the nozzle passes over two separate procedures. The first part includes the selection of location vectors from the parametric space and consequential optimization of vector sequence in order to minimize the overall deformation defined as follows

where $N=$ no. of shapes and $i=1,2, \ldots, N$,

$$
D_{\text {tot }}=\sum_{i=1}^{i=N} D\left(c_{i}, c_{i+1}\right)
$$

$$
D_{\text {tot }} \sum_{i=1}^{i=N} D\left(c_{i}, c_{i+1}\right)
$$

where $D\left(c_{i}, c_{i+1}\right)$ is the Euclidean distance between vectors (shapes) in $\mathrm{R}^{2}$ defined as follows 


$$
D\left(c, c^{\prime}\right)=\sqrt{\left(w_{1}-w_{1}^{\prime}\right)^{2}+\cdots\left(w_{n}-w_{n}^{\prime}\right)^{2}}
$$

and $c, c^{\prime}$ are two data series (series of y-coordinate vectors) as follows

$$
c=\left\langle w_{1}, w_{2}, \ldots, w_{n}\right\rangle c^{\prime}=\left\langle w_{1}^{\prime}, w_{2}^{\prime}, \ldots, w_{n}^{\prime}\right\rangle
$$

$w, w^{\prime}$ represent y-coordinate of nozzle shape consistently sampled by defined $\Delta \mathrm{x}$. Flow, turbulence, and status variables in control volume and their fluxes are preserved for the newly formed mesh from the previous shape. With the acceptably small boundary deformation, the complete converging process takes only an incomparable small number of steps when comparing to "zero" initialization. To satisfy the condition of acceptable small boundary deformation, the shortest distance $\mathrm{D}_{\text {tot }}$ needs to be found out. This problem can be associated as a well-known Travelling Salesman Problem. The decision version of TSP belongs to NP-complete problems. The only approach able to improve the objective is a heuristic algorithm. One of the most sufficient is the Genetic algorithm presented firstly by Holand in [5]. We decided to use the parameter based coding of individuals where the chromosome gets a value of parameters as a real number. The selection of individuals for further evolution is based on the fitness proportionate selection with its modification: stochastic universal sampling. The recombination of individuals is provided by Edge recombination crossover (ERX). The operator of mutation is randomly applied to a selected pair of parameters by bilateral exchange. A more detailed view can be taken in Breitkopf and Coelho [6] and Hynek [7].

A shape-based similarity approach is originally developed by Yanagisawa et $a l$. in [8] for the optimization of the mobile object trajectory although applicable even in the shape - based similarity query.

The second part of the optimization procedure includes CFD simulation of flow over the whole shape sequence of designed nozzles. The actual shape mutates towards the subsequent geometry until the final shape is met.

\subsection{Results of optimization at steady state}

As a first, the seeking for optimized sequence of shape deformation was performed. Evolution of overal deformation from the origanate to finalized shape sampled by $\Delta x=0.1 \mathrm{~mm}$ are in the Figure 7 . The optimization of the shape sequence permutation led to the sequence gaining half of origal value. The best chanel shape sequence found with $D_{\text {tot }}=3383 \mathrm{~mm}$ is used to explore the optimization space. The best variant achieves about $9 \%$ les of vapour volume fraction at the exit of domain than the originally cylindrical channel. Despite an achieved improvement, possible limitations of the approach are found because the best variant comes with the highest exit diameter (coordinate $\mathrm{y}_{2}$ ) available from limited optimization space. For further optimization, at least the upper boundary of coordinate $\mathrm{y}_{2}$ should be increased. There are contours of phase volume rate in the Figure 8 for best variant found. The location of pressure minima corresponds to originate shape, but the pressure jump is distributed within larger area of channel exit and channel throat. Consequently the local pressure minimum is restricted. 


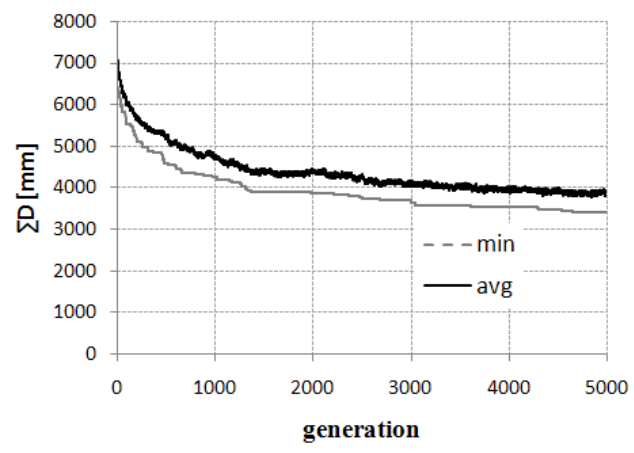

Figure 7: Evolution of average and minimal deformational path of shape sequences in the course of evolutionary algorithm.

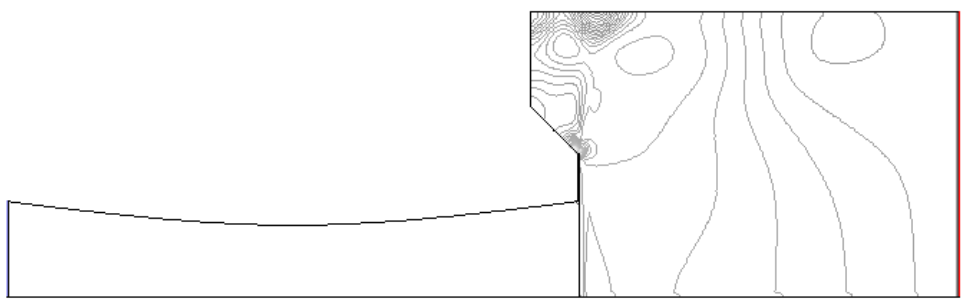

Figure 8: Isolines of vapour volume fraction in the best variant found by optimization. $0-0.5 \mathrm{~m}^{3}$ vapour $/ \mathrm{m}^{3}$ oil.

\section{Conclusions}

The simplified method which enables the unsteady simulation of oil - chocking plate interaction is developed and tested. This method was implemented into commercial FVM code to expand its abilities beyond standards. Numerous techniques used to stabilize the coupled simulations are exposed. Dynamic behaviour of plate lift is than solved and cavitation phenomenon observed. We have applied the shape-similarity-based optimization procedure to reduce the cavitation within the damping unite at steady state previously defined. The best by-pass channel shape and plate stiffness combination comes with the vapour volume rate limited by $15 \%$ when compare to original value.

\section{Nomenclature}

$d \vec{r}, d \vec{r}[0] \quad$ vector from boundary face centroids to wall adjacent cell centroids $\mathrm{k} \quad$ absorber coefficient [-]

$\mathrm{f} \quad$ frequency of movement $[\mathrm{Hz}]$

$\mathrm{r}_{\mathrm{j}} \quad$ radial coordinate of node $\mathrm{j}[\mathrm{mm}]$ 


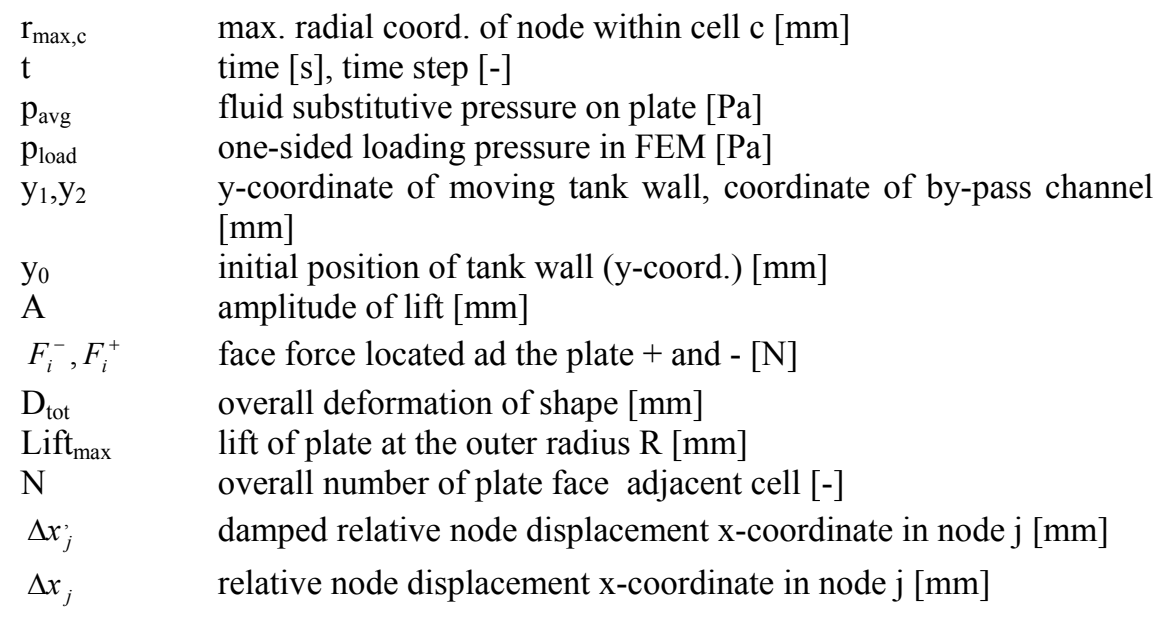

\section{References}

[1] Fluent Inc. Fluent user documentation. Lebanon : s.n., 2006.

[2] Kolář J., Dvořák V. Aerodynamic optimization of supersonic nozzle exploiting dynamic meshes and sentivity analysis, In the 2nd International Conference On Engineerig Optimization 2010 Lisboa, Portugal, Istituto Superio Técnico, 2010. ISBN 978-989-96264-3-0.

[3] Dvořák V., Kolář J. Shape optimization of supersonic ejectors with several primary nozzles. Lisboa, Portugal : In the 2nd International Conference on Engineering Optimization, 6.-9. september 2010. ISBN 978-989-96264-3-0.

[4] Versteeg H. K., Malalasekera W. An introduction to fluid dynamics. Harlow : Pearsons education limited, 2007. ISBN-978-0-13-127498-3.

[5] Holand, H J. Adaption in natural and artificial systems. Ann Arbor, Michigan: The University of Michigan Press, 1975.

[6] Breitkopf P., Coelho R. F. Multidisciplinary design optimization in computational mechanics. s.1. : Wiley-ISTE, 2010. ISBN-10 1848211384.

[7] Hynek J. Genetic algorithms and genetic programing (in Czech). Prague : Grada Publishing ,a. s., 2008. ISBN 978-80-247-2695-3.

[8] Yanagisawa Y., Akahani J., Satoh T. Shape-based similarity query for trajectories of mobile objects. Taipei : Mobile Data Management p. 63-77, 2003. ISSN 0302-9743. 